Web-based monitoring tools for Resistive Plate Chambers in the CMS experiment at CERN

This content has been downloaded from IOPscience. Please scroll down to see the full text.

2014 JINST 9 C10031

(http://iopscience.iop.org/1748-0221/9/10/C10031)

View the table of contents for this issue, or go to the journal homepage for more

Download details:

IP Address: 157.193.98.234

This content was downloaded on 11/06/2015 at $14: 03$

Please note that terms and conditions apply. 


\section{Web-based monitoring tools for Resistive Plate Chambers in the CMS experiment at CERN}

M.S. Kim, ${ }^{u}, 1$ Y. Ban, ${ }^{a}$ J. Cai, ${ }^{a}{ }^{\text {Q }}$. Li, ${ }^{a}$ S. Liu,,${ }^{a}$ S. Qian,,${ }^{a}$ D. Wang,,${ }^{a}$ Z. Xu, ${ }^{a}$ F. Zhang, ${ }^{a}$ Y. Choi, ${ }^{b}$ D. Kim, ${ }^{b}$ J. Goh, ${ }^{b}$ S. Choi, ${ }^{c}$ B. Hong, ${ }^{c}$ J.W. Kang, ${ }^{c}$ M. Kang, ${ }^{c}$ J.H. Kwon, ${ }^{c}$ K.S. Lee, ${ }^{c}$ S.K. Lee, ${ }^{c}$ S.K. Park, ${ }^{c}$ L.M. Pant, ${ }^{d}$ A.K. Mohanty, ${ }^{d}$ R. Chudasama, ${ }^{d}$ J.B. Singh, ${ }^{e}$ V. Bhatnagar, ${ }^{e}$ A. Mehta, ${ }^{e}$ R. Kumar, ${ }^{e}$ S. Cauwenbergh, ${ }^{f}$ S. Costantini, ${ }^{f}$ A. Cimmino, ${ }^{f}$ S. Crucy, ${ }^{f}$ A. Fagot,${ }^{f}$ G. Garcia,${ }^{f}$ A. Ocampo,,${ }^{f}$ D. Poyraz,${ }^{f}$ S. Salva,${ }^{f}$ F. Thyssen, ${ }^{f}$ M. Tytgat, ${ }^{f}$ N. Zaganidis, ${ }^{f}$ W.V. Doninck, ${ }^{g}$ A. Cabrera,${ }^{h}$ L. Chaparro, ${ }^{h}$ J.P. Gomez, ${ }^{h}$ B. Gomez, ${ }^{h}$ J.C. Sanabria, ${ }^{h}$ C. Avila, ${ }^{h}$ A. Ahmad, ${ }^{i}$ S. Muhammad, ${ }^{i}$ M. Shoaib, ${ }^{i}$ H. Hoorani, ${ }^{i}$ I. Awan,,${ }^{i}$ I. Ali, ${ }^{i}$ W. Ahmed, ${ }^{i}$ M.l. Asghar, ${ }^{i}$ H. Shahzad, ${ }^{i}$ A. Sayed,${ }^{j}$ A. Ibrahim, ${ }^{j}$ S. Aly, ${ }^{j}$ Y. Assran, ${ }^{j}$ A. Radi, ${ }^{j}$ T. Elkafrawy, ${ }^{j}$ A. Sharma, ${ }^{k}$ S. Colafranceschi, ${ }^{k}$ M. Abbrescia, ${ }^{l}$ C. Calabria, ${ }^{l}$ A. Colaleo, ${ }^{l}$ G. laselli, ${ }^{l}$ F. Loddo, ${ }^{l}$ M. Maggi, ${ }^{l}$ S. Nuzzo, ${ }^{l}$ G. Pugliese, ${ }^{l}$ R. Radogna,${ }^{l}$ R. Venditti, ${ }^{l}$ P. Verwillingen, ${ }^{l}$ L. Benussi, ${ }^{m}$ S. Bianco, ${ }^{m}$ D. Piccolo, ${ }^{m}$ P. Paolucci, ${ }^{n}$ S. Buontempo, ${ }^{n}$ N. Cavallo, ${ }^{n}$ M. Merola, ${ }^{n}$ F. Fabozzi, ${ }^{n}$ O.M. Iorio, ${ }^{n}$ A. Braghieri, ${ }^{o}$ P. Montagna, ${ }^{o}$ C. Riccardi, ${ }^{o}$ P. Salvini, ${ }^{o}$ P. Vitulo, ${ }^{o}$ I. Vai, ${ }^{o}$ A. Magnani, ${ }^{o}$ A. Dimitrov, ${ }^{p}$ L. Litov, ${ }^{p}$ B. Pavlov, ${ }^{p}$ P. Petkov, ${ }^{p}$ A. Aleksandrov, ${ }^{q}$ V. Genchev, ${ }^{q}$ P. laydjiev, ${ }^{q}$ M. Rodozov, ${ }^{q}$ G. Sultanov, ${ }^{q}$ M. Vutova, ${ }^{q}$ S. Stoykova, ${ }^{q}$ R. Hadjiiska, ${ }^{q}$ H.S. Ibargüen, ${ }^{r}$ M.I.P. Morales, ${ }^{r}$ S.C. Bernardino, ${ }^{r}$ I. Bagaturia, ${ }^{s}$ Z. Tsamalaidze ${ }^{s}$ and I. Crotty ${ }^{t}$ on behalf of the CMS collaboration

\footnotetext{
${ }^{a}$ School of Physics, Peking University, CN-100 871 Beijing, China

${ }^{b}$ Department of Physics, SungKyunKwan University,

2066, Seobu-ro, Jangahn-gu, Suwon, Gyeonggi-do, Republic of Korea

${ }^{c}$ Department of Physics and Korea Detector Laboratory, Korea University,

Aman-dong 5-ga, Sungbuk-gu, Seoul, Republic of Korea

${ }^{d}$ Electronics Division, BARC, Trombay, Mumbai 400 085, India

${ }^{e}$ Department of Physics, Panjab University, Chandigarh Mandir 160 014, India
}

\footnotetext{
${ }^{1}$ Corresponding author.
} 
${ }^{f}$ Department of Physics and Astronomy, Ghent University, Proeftuinstraat 86, BE-9000 Ghent, Belgium

${ }^{g} V U B$, Dienst Elementaire Deeltjes, Pleinlaan 2, BE-1050 Brussel, Belgium

${ }^{h}$ Universidad de Los Andes, Apartado Aéreo 4976, Carrera 1E, no. 18A 10, CO-Bogotá, Colombia

${ }^{i}$ Islamabad-NCP, Quaid-I-Azam University, Islamabad 44000, Pakistan

${ }^{j}$ Academy of Scientific Research and Technology of the Arab Republic of Egypt,

101 Sharia Kasr El-Ain, Cairo, Egypt

${ }^{k}$ CERN, CH-1211 Geneva 23, Switzerland

${ }^{l}$ Universita e INFN, Sezione di Bari, Via Orabona 4, IT-70126 Bari, Italy

${ }^{m}$ INFN, Laboratori Nazionali di Frascati, PO Box 13, Via Enrico Fermi 40, IT-00044 Frascati, Italy

${ }^{n}$ Universita e INFN, Sezione di Napoli,

Complesso Univ. Monte S. Angelo, Via Cintia, IT-80126 Napoli, Italy

${ }^{\circ}$ Universita e INFN, Sezione di Pavia, Via Bassi 6, IT-Pavia, Italy

${ }^{p}$ Faculty of Physics, Atomic Physics Department, University of Sofia,

5, James Bourchier Boulevard, BG-1164 Sofia, Bulgaria

${ }^{q}$ Inst. for Nucl. Res. and Nucl. Energy, Bulgarian Academy of Sciences,

Tzarigradsko shaussee Boulevard 72, BG-1784 Sofia, Bulgaria

${ }^{r}$ Benemerita Universidad Autonoma de Puebla, Av. San Claudio y 18 sur, edif. 111A,

Ciudad Universitaria, Col. San Manuel, Puebla, Pue. C.P. 72570, Mexico

${ }^{s}$ Tbilisi-IHEPI, Tbilisi State University, University Street 9, GE-0186 Tbilisi, Georgia

${ }^{t}$ Department of Physics, University of Wisconsin, 1150 University Avenue, Madison, WI 53706, U.S.A.

${ }^{u}$ Department of Physics, Kyungpook National University,

80 Daehakro, Bukgu, Daegu, 702-701, Republic of Korea

E-mail: minsuk@cern.ch

AbSTRACT: The Resistive Plate Chambers (RPC) are used in the CMS experiment at the trigger level and also in the standard offline muon reconstruction. In order to guarantee the quality of the data collected and to monitor online the detector performance, a set of tools has been developed in CMS which is heavily used in the RPC system. The Web-based monitoring (WBM) is a set of java servlets that allows users to check the performance of the hardware during data taking, providing distributions and history plots of all the parameters. The functionalities of the RPC WBM monitoring tools are presented along with studies of the detector performance as a function of growing luminosity and environmental conditions that are tracked over time.

KEYWORDS: Muon spectrometers; Resistive-plate chambers 


\section{Contents}

1 Introduction 1

2 The CMS RPC system 1

3 WBM monitoring tools for RPC 2

4 Conclusions 4

\section{Introduction}

The World Wide Web was invented in 1989 at CERN near Geneva, Switzerland. Since that time, the Web has been used as a tool in High Energy Physics (HEP) to exchange data and results. In 25 years after the first connection was established, millions of computers have been connected together through the Internet and HEP experiments have continued to enlarge this favorite collaborative tool.

Monitoring in the CMS experiment [1] is a demanding task with the growth in data size and complexity. The amount of people physically present in the control rooms of the experiment is limited and there is a need for remote monitoring so that the shift takers and experts can detect, diagnose and fix problems from remote locations.

Monitoring is a key component in the effort to achieve efficient and high quality data taking. The data quality monitoring and certification systems from online data taking to delivering certified data sets for physics analyses, release validation and offline re-reconstruction activities at Tier-1 sites are well described in ref. [2]. The Web-based monitoring (WBM) has the goal to provide a suite of tools that is easy to use, accessible through firewalls, secure, flexible, and maintainable as described in ref. [3, 4].

In this paper, we present the functionality of the WBM monitoring tools for RPC, employed by shift crew members, detector subsystem experts, operation coordinators, and those performing data analysis.

\section{The CMS RPC system}

The RPCs are bakelite-based, double-gap detectors with bulk resistivity ranges 1-6 $\cdot 10^{10} \Omega \cdot \mathrm{cm}$. Each gap is composed by two parallel electrodes, each of $2 \mathrm{~mm}$ thickness, and a $2 \mathrm{~mm}$ wide gas gap, and operated with a gas mixture in avalanche mode to safeguard the time resolution at high rates $\left(\sim 1 \mathrm{kHz} / \mathrm{cm}^{2}\right)$. The water vapor is added to keep its relative humidity around $45 \%$ so that the bakelite resistivity remains constant, avoiding a degradation of performance under high background conditions. Readout copper strips along the beam direction are sandwiched between the two gaps, with an independent electronic channel per strip, segmented in pseudo-rapidity $\eta$. The total readout system of the RPC detector consists of $\sim 120000$ channels in about $4000 \mathrm{~m}^{2}$ of active area. 


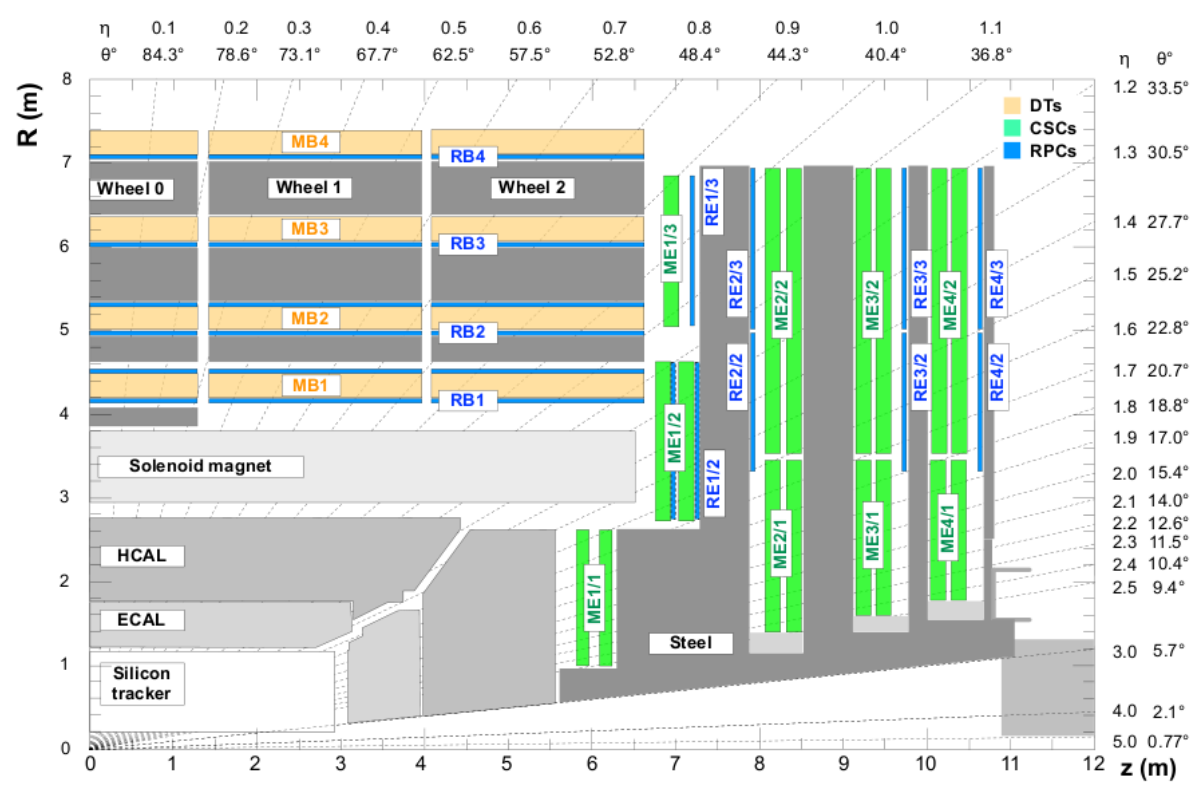

Figure 1. Layout of one quadrant of the CMS detector. Muon stations are placed in the steel return yoke of the magnet (gray). The four DT stations in the barrel (yellow), the four CSC disks in the endcap (green), and the RPCs in both barrel and endcap regions (blue) are shown. The fourth disks of new CSCs and RPCs (ME4 and RE4 for $1.2<|\eta|<1.8$ ) are installed during the first Long Shutdown at the LHC in 2013-2014.

The CMS muon system consists of three independent and complementary sub-systems: Drift Tubes (DT) and Cathode Strip Chambers (CSC) in the barrel and endcap regions respectively, and RPCs in both the regions. A schematic view of the CMS detector in the R-z plane is shown in figure 1. Along the $\mathrm{z}$ axis, the DTs and RPCs in the barrel are divided into 5 wheels, with Wheel 0 in the center of the detector (i.e., $\mathrm{z}=0$ ) and wheels $\mathrm{W} \pm 1$ and $\mathrm{W} \pm 2$ in the $\pm \mathrm{z}$ directions. The endcap region is composed of four iron disks on each side along $\mathrm{z}$. A station is an assembly of chambers around a fixed value of $\mathrm{R}$ in the barrel and $\mathrm{z}$ in the endcaps. On each side of the CMS detector, three of the endcap yoke disks hold a total of four CSC planes and RPC planes, where each disk is divided into 36 sectors with up to 3 radial rings per sector. Every chamber is then divided from the readout point of view into 2 or $3 \eta$ partitions called rolls. A more detailed description of the CMS RPC detector can be found in ref. [5].

During the first three-year LHC running period, the fourth endcap stations remained largely uninstrumented. CSCs were only installed in the very forward region $(1.8<|\eta|<2.4)$, and RPCs were missing completely and covered only the first three endcap disks $(|\eta|<1.6)$. During the first long shutdown (LS1) for the upgrades and repairs in 2013-2014, these fourth endcap stations were instrumented further with new CSCs (ME4) and RPCs (RE4) up to $|\eta|=1.8$. There are a total of 912 (1056) RPC chambers before (after) LS1; 480 in the barrel and 432 (576) in the endcaps.

\section{WBM monitoring tools for RPC}

The RPC WBM is part of the WBM services which provides monitoring of both real-time and archival data. Sources of information, which can be obtained via specialized hardware, are the CMS experiment and the LHC accelerator at CERN. The WBM server runs an Apache daemon [6] 

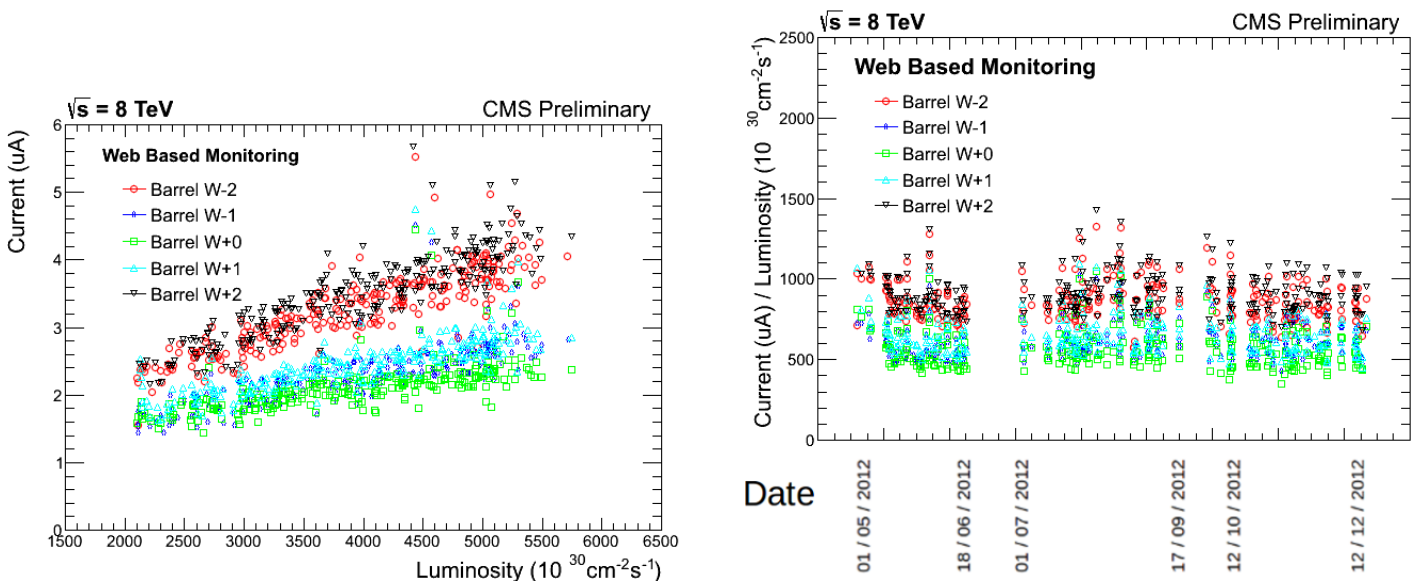

Figure 2. The RPC chamber current for each wheel in the barrel as a function of growing instantaneous luminosity (left) and the current divided by luminosity as a function of time (right). Each gap in time indicates a short maintenance period of the LHC.

to respond to HTTP requests and most of the user interactive work is handled through Apache Tomcat [7], using a java servlet architecture. The ROOT [8] package is used for historical plotting on the hardware performance and data manipulation. Many of the pages use SQL (Structured Query Language) to communicate with the CMS online database.

The main parameters for the RPC operation can be monitored and controlled by the Detector Control System. Their values were saved in a proper database and used offline to study the system stability. The parameters of the RPC system such as high voltage, low voltage, current, temperatures, pressure, gas flow, noise, status of readout strips, and the other environmental variables available in the database can be also monitored through the RPC WBM. These functionalities of the WBM system allow both the experts and shift takers to have a clear idea of the detector performance during a cosmic or collision run, or the particular running period. The ability of the RPC WBM to allow the access and handling of data at the roll is crucial for this. The monitoring result can be averaged over all sectors in each wheel and station. Furthermore, it can be averaged separately for wheels and disks, or barrel and endcaps.

As an example of the plots that can be obtained using the RPC WBM monitoring tools, figure 2 shows the RPC chamber current in the barrel as a function of growing instantaneous luminosity and the same current divided by instantaneous luminosity as a function of time during the 2012 LHC run at $8 \mathrm{TeV}$. For every wheel in the barrel, the linear correlation of the chamber current and the delivered instantaneous luminosity can be clearly seen. Differences are observed for the wheels that are located at different positions along the beam direction z. A good linearity is found between the current, divided by the luminosity, and time.

Similarly, the measured rate has such a linear correlation as shown in figure 3 for every wheel and averaged on wheels and disks. An asymmetry is found between the wheels $\mathrm{W} \pm 2$, which is under investigation during the first Long Shutdown at the LHC in 2013-2014. Figure 4 shows the time-independent rate normalized to the luminosity. Sources of these main parameters are particles coming from the interaction point or neutron or non-collision beam-related background. 

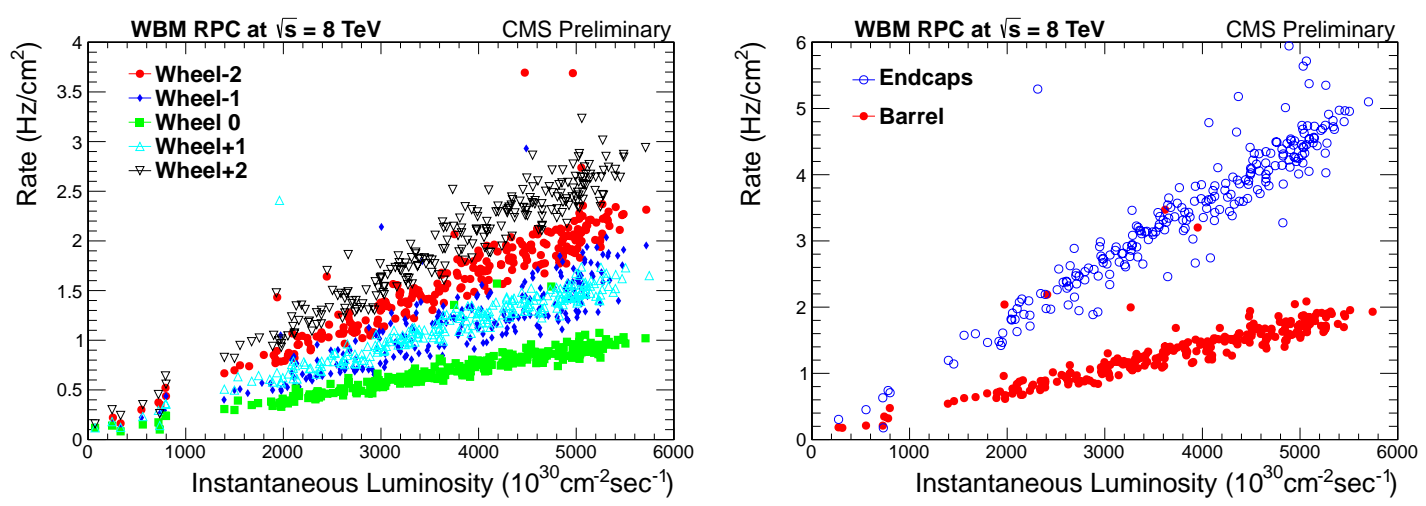

Figure 3. The measured rate for each wheel in the barrel (left) and averaged on wheels and disks (right) as a function of growing instantaneous luminosity.
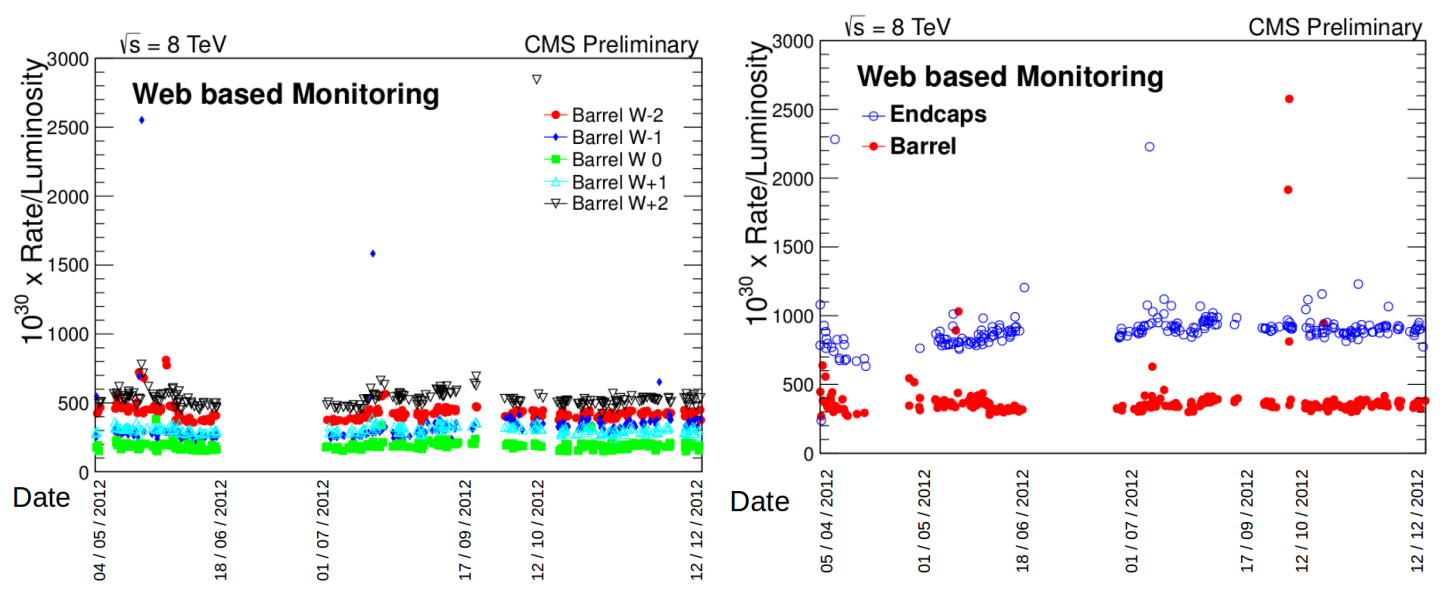

Figure 4. The measured rate for each wheel in the barrel (left) and averaged on wheels and disks (right) divided by luminosity as a function of time. Each gap in time indicates a short maintenance period of the LHC.

The RPC chamber current and rate are the most important information about LHC and RPC performance for the data certification that the shift crew must provide as soon as the data are available. After having verified the detector performance using the WBM monitoring tools, the data integrity and quality can be easily checked with the data quality monitoring service.

\section{Conclusions}

The RPC WBM monitoring tools has been developed and used for studies of the detector performance during the $2012 \mathrm{LHC}$ run at $8 \mathrm{TeV}$. The web-based nature of the service can provide users around the world with useful information through a simple web page, leading the challenge on a global collaboration to monitor the performance of the LHC and the CMS detector and to certify the data for physics analyses. 


\section{Acknowledgments}

We congratulate our colleagues in the CERN accelerator departments for the excellent performance of the LHC and thank the technical and administrative staffs at CERN and at other CMS institutes for their contributions to the success of the CMS effort. In addition, we gratefully acknowledge the computing centres and personnel of the Worldwide LHC Computing Grid for delivering so effectively the computing infrastructure essential to our analyses. Finally, we acknowledge the enduring support for the construction and operation of the LHC and the CMS detector provided by the following funding agencies: BMWFW and FWF (Austria); FNRS and FWO (Belgium); CNPq, CAPES, FAPERJ, and FAPESP (Brazil); MES (Bulgaria); CERN; CAS, MoST, and NSFC (China); COLCIENCIAS (Colombia); MSES and CSF (Croatia); RPF (Cyprus); MoER, ERC IUT and ERDF (Estonia); Academy of Finland, MEC, and HIP (Finland); CEA and CNRS/IN2P3 (France); BMBF, DFG, and HGF (Germany); GSRT (Greece); OTKA and NIH (Hungary); DAE and DST (India); IPM (Iran); SFI (Ireland); INFN (Italy); NRF and WCU (Republic of Korea); LAS (Lithuania); MOE and UM (Malaysia); CINVESTAV, CONACYT, SEP, and UASLP-FAI (Mexico); MBIE (New Zealand); PAEC (Pakistan); MSHE and NSC (Poland); FCT (Portugal); JINR (Dubna); MON, RosAtom, RAS and RFBR (Russia); MESTD (Serbia); SEIDI and CPAN (Spain); Swiss Funding Agencies (Switzerland); MST (Taipei); ThEPCenter, IPST, STAR and NSTDA (Thailand); TUBITAK and TAEK (Turkey); NASU and SFFR (Ukraine); STFC (United Kingdom); DOE and NSF (U.S.A.).

\section{References}

[1] CMS collaboration, The CMS experiment at the CERN LHC, 2008 JINST 3 S08004.

[2] L. Tuura et al., CMS data quality monitoring: Systems and experiences, J. Phys. Conf. Ser. 219 (2010) 072020.

[3] W. Badgett et al., Web based monitoring in the CMS experiment at CERN, J. Phys. Conf. Ser. 331 (2011) 022025.

[4] W. Badgett et al., New Developments in Web Based Monitoring at the CMS experiment, J. Phys. Conf. Ser. 396 (2012) 062002.

[5] CMS collaboration, Performance of CMS muon detector in proton-proton collisions at $\sqrt{s}=7 \mathrm{TeV}$ at the LHC, 2013 JINST 8 P11002 [arXiv: 1306.6905v2].

[6] http://httpd.apache.org/.

[7] http://tomcat.apache.org/.

[8] http://root.cern.ch/. 